\title{
Identification and evaluation of metastasis-related proteins, oxysterol binding protein-like 5 and calumenin, in lung tumors
}

\author{
KAZUYA NAGANO $^{1 *}$, SUNAO IMAI $^{1^{*}}$, XILULI ZHAO $^{1 *}$, TAKUYA YAMASHITA ${ }^{1}$, YASUO YOSHIOKA ${ }^{1-3}$, \\ YASUHIRO ABE ${ }^{1}$, YOHEI MUKAI ${ }^{1}$, HARUHIKO KAMADA $^{1,3}$, SHINSAKU NAKAGAWA ${ }^{2,3}$, \\ YASUO TSUTSUMI ${ }^{1-3}$ and SHIN-ICHI TSUNODA ${ }^{1-3}$ \\ ${ }^{1}$ Laboratory of Biopharmaceutical Research, National Institute of Biomedical Innovation, Ibaraki, Osaka 567-0085; \\ ${ }^{2}$ Graduate School of Pharmaceutical Sciences, ${ }^{3}$ The Center of Advanced Medical Engineering \\ and Informatics, Osaka University, Suita, Osaka 565-0871, Japan
}

Received February 5, 2015; Accepted March 18, 2015

DOI: $10.3892 /$ ijo.2015.3000

\begin{abstract}
Metastasis is an important prognosis factor in lung cancer, therefore, it is imperative to identify target molecules and elucidate molecular mechanism of metastasis for developing new therapeutics and diagnosis methods. We searched for metastasis-related proteins by utilizing a novel antibody proteome technology developed in our laboratory that facilitated efficient screening of useful target proteins. Two-dimensional differential in-gel electrophoresis (2D-DIGE) analysis identified sixteen proteins, which were highly expressed in metastatic lung cancer cells, as protein candidates. Monoclonal single-chain variable fragments (scFvs) binding to candidates were isolated from a scFv-displaying phage library by affinity selection. Tissue microarray analysis of scFvs binding to candidates revealed that oxysterol binding protein-like 5 (OSBPL5) and calumenin (CALU) were expressed at a significantly higher levels in the lung tissues of metastasis-positive cases than that in the metastasis-negative cases (OSBPL5; $\mathrm{p}=0.0156$, CALU; $\mathrm{p}=0.0055$ ). Furthermore, $80 \%$ of OSBPL5 and CALU double-positive cases were positive for lymph node metastasis. Consistent with these observations, overexpression of OSBPL5 and CALU promoted invasiveness of lung cancer
\end{abstract}

Correspondence to: Dr Shin-Ichi Tsunoda, Laboratory of Biopharmaceutical Research, National Institute of Biomedical Innovation, 7-6-8 Saito-Asagi, Ibaraki, Osaka 567-0085, Japan

E-mail: tsunoda@nibio.go.jp

${ }^{*}$ Contributed equally

Abbreviations: OSBPL5, oxysterol binding protein-like 5; CALU, calumenin; scFv, single-chain variable fragment; TMA, tissue microarray; 2D-DIGE, two-dimensional differential in-gel electrophoresis; MS, mass spectrometry; TBST, Tris-buffered saline containing Tween-20; KDR, kinase insert domain receptor; TNFR1, tumor necrosis factor receptor 1

Key words: metastasis, lung cancer, antibody proteomics technology, oxysterol binding protein-like 5 , calumenin cells. Conversely, knockdown of these proteins using respective siRNAs reversed the invasiveness of the lung cancer cells. Moreover, these proteins were expressed in lung tumor tissues, but not in normal lung tissues. In conclusion, OSBPL5 and CALU are related to metastatic potential of lung cancer cells, and they could be useful targets for cancer diagnosis and also for development of drugs against metastasis.

\section{Introduction}

Lung cancer is the leading cause of cancer-related deaths in the world, accounting for $\sim 17.6 \%$ of all deaths from cancer, 5-year survival rate for which is only $8.9-15 \%$ (1). Particularly, metastasis is one of the poorest prognosis factors in lung cancer, and is the main cause that leads to treatment failure and death (2). Thus, it is imperative to overcome metastasis in order to decrease lung cancer related mortality. Several mechanistic studies have revealed that various proteases $(3,4)$, chaperones $(5,6)$, epithelial-mesenchymal transition (EMT) $(7,8)$ and lipid metabolism $(9,10)$ may be associated with metastasis. However, these results are not exhaustive, and no promising candidate has been identified so far for the accurate diagnosis, prediction and regulation of metastasis in clinical settings.

In recent years, proteomic analysis has become a preferred method for seeking diagnostic markers or drug targets (11). However, in general, a lot of proteins are differentiallyexpressed in disease samples. Thus, the rate limiting step is to select the most useful proteins from many differentiallyexpressed proteins. In order to circumvent this problem, we have developed an 'antibody proteomics technology' to accelerate identifying proteins which would be useful for elucidating the molecular mechanism of metastasis and developing accurate diagnosis or effective therapy as well for metastasis (12). This technology enabled us to comprehensively and rapidly generate monoclonal antibodies against candidate proteins, including unknown proteins, for which no commercially produced antibodies are available, by screening a single-chain variable fragment ( $\mathrm{scFv}$ ) phage display library using small amount of proteins, which were directly extracted from a 2-dimensional gel used for the proteome analysis. 
Therefore, by immunostaining a tissue microarray (TMA), a glass slide containing many clinical samples (such as tumor and normal tissues) and clinical information [such as age, gender, clinical stage and lymph node (LN) metastasis], it is possible to validate each candidate protein by analyzing the correlation between the expression profile of each candidate protein and clinical information (13-15). As reported previously, we have successfully used this technology to quickly identify useful breast cancer-related proteins, thus suggesting its practical usefulness (16-18).

We applied the above described technology to lung cancer cells with different metastatic abilities for subsequent identification of lung cancer metastasis-related proteins, which could be used for elucidating the molecular mechanism of metastasis, and developing novel diagnostic methods and therapies for metastasis.

\section{Materials and methods}

Cell cultures. The human lung cancer cell lines, RERF-LC-KJ, RERF-LC-MS, HARA and HARA-B, were purchased from the Japanese Collection of Research Bioresources Cell Bank (Osaka, Japan). All cells were cultured in RPMI-1640 medium with $10 \%$ fetal calf serum (FCS) at $37^{\circ} \mathrm{C}$ in a humidified atmosphere of $5 \% \mathrm{CO}_{2}$.

Two-dimensional differential in-gel electrophoresis (2D-DIGE) analysis. RERF-LC-MS and RERF-LC-KJ cell lysates were labelled with the $\mathrm{Cy} 3$ and $\mathrm{Cy} 5$ protein labeling dyes (GE Healthcare Bio-Sciences AB, Uppsala, Sweden), respectively. The labelled samples were mixed and applied to a $24-\mathrm{cm}$ immobilized $\mathrm{pH}$ gradient gel strip (IPG-strip $\mathrm{pH}$ 4.0-7.0) for first dimension separation. For the second dimension separation, the IPG-strips were placed on the top of the SDS-PAGE gels. After electrophoresis, gels were scanned with a laser fluoroimager (Typhoon Trio, GE Healthcare Bio-Sciences AB, Uppsala, Sweden). Quantitative analysis of protein spots was carried out with Decyder-DIA software (GE Healthcare Bio-Sciences AB). The samples for the spotpicking gel were prepared without labelling. The spot-picking gel was scanned after staining with deep purple total protein stain reagent (GE Healthcare Bio-Sciences AB). The antigen spots of interest were picked using an Ettan Spot Picker (GE Healthcare Bio-Sciences AB). Proteins were extracted by solubilizing the picked gel pieces using $88 \mathrm{mM}$ sodium periodide for nitrocellulose panning experiment.

Protein identification by mass spectrometry analysis. Picked gel pieces were in-gel digested with trypsin overnight. The digested peptides were dried and resuspended in $10 \mu \mathrm{l}$ of $0.1 \%$ trifluoroacetic acid, following which they were purified using ZipTip $\mu \mathrm{C}_{18}$ pipette tips (EMD Millipore, Billerica, MA, USA). The digested peptides were analyzed by matrix-assisted laser desorption ionization time-of-flight mass spectrometry (MALDI-TOF/MS; AutoflexII, Bruker Daltonics Inc., Billerica, MA, USA). Peptide mass fingerprints were used for searching public protein primary sequence databases to identify proteins. The Mascot search engine (http://www. matrixscience.com) was initially used to query the entire theoretical tryptic peptide.
Isolation of monoclonal antibodies by panning. To generate monoclonal antibodies, panning of the $\mathrm{scFv}$ phage display library was performed using nitrocellulose membrane blots as previously described (12). Briefly, a portion of each protein extracted from the 2D-DIGE spots was immobilized onto a nitrocellulose membrane using the Bio-Dot Microfiltration apparatus (Bio-Rad Laboratories, Hercules, CA, USA), and these were then incubated with the blocking solution (10\% skimmed milk, 25\% glycerol) for $2 \mathrm{~h}$. The non-immune scFv phage display library (19) was applied to each well of the Bio-Dot Microfiltration apparatus $\left(10^{12} \mathrm{CFU} /\right.$ well $)$. After 2-3-h incubation, each well was washed ten times with Tris-buffered saline containing $0.05 \%$ Tween-20 (TBST). Bound phage was then eluted with $100 \mathrm{mM}$ triethylamine. The eluted phage was used to infect log phase E. coli TG1 cells and cells were grown for $1 \mathrm{~h}$ at $37^{\circ} \mathrm{C}$. Output phage titer was measured by counting the number of infected cells on Petrifilm (3M Corporate, St. Paul, MN, USA). The panning cycle was repeated four times.

Phage dot blot ELISA. For each identified target protein, 30 individual phage-infected TG1 clones were picked and grown separately to propagate phages, which were then purified by precipitation with polyethylene glycol. The purified phages $\left(10^{12} \mathrm{CFU} /\right.$ well) were incubated with the respective target proteins, which were extracted from the 2D-DIGE protein spots and immobilized using the Bio-Dot Microfiltration apparatus (Bio-Rad Laboratories) as described above. Phages bound to each target protein were visualized using an HRP-conjugated anti-M13 monoclonal antibody (GE Healthcare Bio-Sciences AB). For specificity assay, human recombinant kinase insert domain receptor (KDR), tumor necrosis factor receptor 1 (TNFR1) (R\&D Systems Inc., Minneapolis, MN, USA), caspase- 8 and importin- $\alpha$ were used as antigens.

Immunohistochemical analysis of TMA. Human lung cancer and normal TMAs (Super BioChips Laboratories, Seoul, Korea) were deparaffinized in xylene and rehydrated in ethanol. After heat-induced epitope retrieval using the Target Retrieval Solution pH 9.0 (Dako, Glostup, Denmark), endogenous peroxidase was blocked with $0.3 \% \mathrm{H}_{2} \mathrm{O}_{2}$ for $5 \mathrm{~min}$. The slides were then incubated with an scFv-displaying phage (1012 CFU/ml) for $30 \mathrm{~min}$. After washing three times with TBST, the slides were incubated for 30 min with Envision ${ }^{+}$ Dual Link (Dako). Finally, the slides were washed three times with TBST and treated with 3,3'-diaminobenzidine, and then counterstained with Mayer's hematoxylin. For statistical analysis, study samples were divided into high and low expression groups based on the following two criteria. In terms of distribution, the percentage of positive cells in a population of all tumor cells was scored as $0(0 \%), 1$ (1-50\%), and 2 (51-100\%). In terms of quantity, the signal intensity was scored as 0 (no signal), 1 (weak), 2 (moderate) or 3 (marked). Cases with a total score of $\geq 3$ were classified into the high expression group.

Manipulation of gene expression by plasmid or siRNA transfection. For overexpression and knockdown of gene expression, cells were transfected with an expression plasmid containing the cDNA of the gene or with a gene-specific siRNA, respectively. Transfection of cells with OSBPL5 and CALU expression 
plasmids (Life Technologies, Carlsbad, CA, USA) was carried out using Lipofectamine LTX (Life Technologies) according to the manufacturer's instructions. Briefly, RERF-LC-MS cells were plated in $100-\mathrm{mm}$ dish. The next day, $15 \mu \mathrm{g}$ of plasmid was mixed with $15 \mu \mathrm{l}$ plus reagent in $3 \mathrm{ml}$ Opti-MEM. After 15-min incubation, $37.5 \mu 1$ lipofectamine LTX was added, incubated for $30 \mathrm{~min}$, and then the DNA-lipofectamine complex was added to the cells. The cells were used in invasion assay after 24-h incubation. Transfection of cells with gene specific siRNA (Qiagen, Montgomery Country, MD, USA) was carried out using HiPerFect Transfection Reagent (Qiagen) according to the manufacturer's instructions. Briefly, RERF-LC-KJ, HARA-B and HARA cells were plated in a $100-\mathrm{mm}$ dish. The next day, $50 \mathrm{nM}$ siRNA was mixed with $40 \mu \mathrm{l}$ HiPerFect Transfection reagent in $3 \mathrm{ml}$ Opti-MEM. After 10-min incubation, the complex was added to the cells. Transfected cells were used in invasion assay after 24-h incubation. The cells treated with only transfection reagents are regarded as mock cells. Expression levels of OSBPL5 and CALU genes in cells, transfected either with the respective cDNA-expression plasmid or with the indicated siRNA, were determined using the RT-PCR method.

Invasion assay. Invasion assay was performed using a 96-well BME cell invasion assay kit (Trevigen Inc., Gaithersburg, MD, USA). The upper chambers of the 96-well cell culture inserts were washed with serum-free medium, coated with $50 \mu \mathrm{l}$ of basal membrane extract (BME) and then dried overnight at $37^{\circ} \mathrm{C}$. One million cells in serum-free media were added to the upper chambers and $150 \mu \mathrm{l}$ of medium containing $10 \%$ FCS was added to the lower chambers. The invasion chambers were kept for $72 \mathrm{~h}$ at $37^{\circ} \mathrm{C}$ in the cell culture incubator. Non-invasive cells on the upper insert membranes were removed by gentle rubbing. Invasive cells on the lower insert membranes were stained with calcein-AM solution, and were assayed by measuring the fluorescence intensity using ARVO MX (Perkin-Elmer, Waltham, MA, USA).

\section{Results}

2D-DIGE analysis and isolation of antibodies against differentially-expressed proteins using non-immune scFv-displaying phage library. In order to identify metastasis-related proteins in lung cancer, we performed 2D-DIGE analysis of lung cancer cells with high LN metastatic potential (RERF-LC-KJ) $(20,21)$ and lung cancer cells with non-metastatic potential (RERF-LC-MS) $(22,23)$. Fig. 1 shows the fluorescent image of a representative $2 \mathrm{D}$-gel containing proteins expressed in these cells. Quantitative analysis identified 15 protein spots whose intensities altered $>2$-fold in RERF-LC-KJ cells compared to in RERF-LC-MS cells. Proteins from these spots were then identified by MALDI-TOF/MS (Table I). Thus, a portion of each extracted protein was immobilized by dot-blotting onto a nitrocellulose membrane and this membrane was used for 4-cycle panning of a non-immune scFv-displaying phage library. The output/input ratio (titer of the recovered phage library after the panning/titer of the library before the panning) was increased as the panning round was repeated (Table II). This elevated output/input ratio indicated the enrichment of antigen-binding $\mathrm{scFv}$ clones. A total of 30 clones (for each

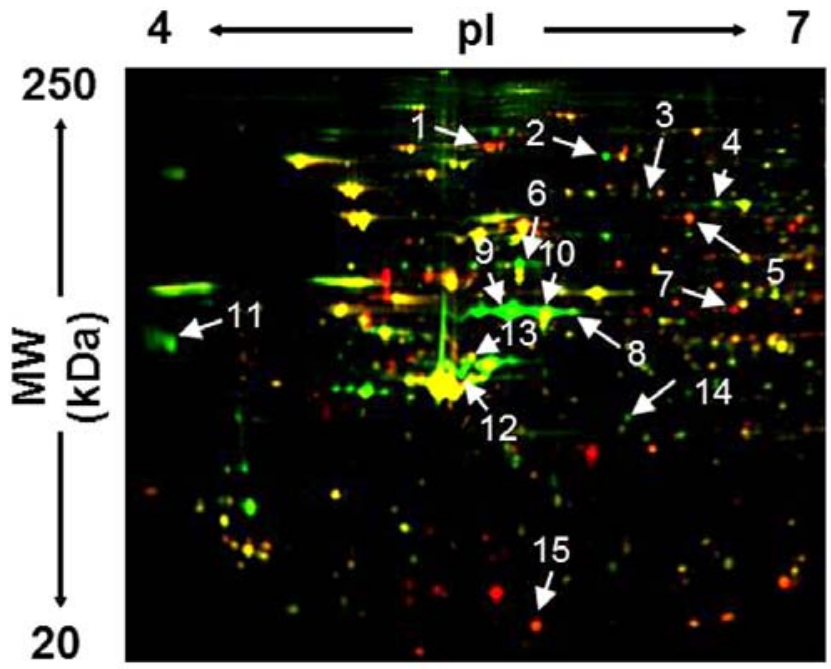

Figure 1.2D-DIGE image of fluorescently labeled proteins from RERF-LC-KJ and RERF-LC-MS cells. Proteins were extracted from RERF-LC-KJ and RERF-LC-MS lung cancer cells with different metastatic potential to LNs. Proteins extracted from these two cells were labeled, respectively, with $\mathrm{Cy} 3$ and Cy5 protein labelling dyes, mixed, and then the mixture was subjected to 2D-electrophoresis. Green spot indicates a protein whose expression is higher in RERF-LC-KJ cells than in RERF-LC-MS cells. Red spot indicates a protein whose expression is lower in RERF-LC-KJ cells than in RERF-LC-MS cells. Yellow spot indicates that the expression level of the protein in RERF-LC-KJ and RERF-LC-MS cells could not be differentiated. The spots marked with arrows are the proteins identified by MALDI-TOF/MS.

target protein) were randomly picked from the fourth panning output and their bindings to respective antigens were verified by phage dot blot ELISA. Results shown in Fig. 2 demonstrated that each one of the 15 target proteins were able to bind to multiple number of $\mathrm{scFv}$ antibody phages. From these positive clones, we selected the ones displaying highest affinities for evaluating their respective specificity. Fig. 3 showed the specificity evaluation results, which demonstrated that all selected scFv clones specifically recognized their respective target proteins, but not KDR, TNFR1, caspase- 8 and importin- $\alpha$, which were used as negative control antigens. Thus, by using the antibody proteomics technology described in this study, we isolated monoclonal antibodies to 15 metastasis-related target proteins and validated their specificity.

TMA analysis. In order to identify and select the metastasisrelated proteins in lung cancer from a large pool of candidate proteins, expression profiles of the identified proteins were determined by TMA analysis using the phage antibodies. The TMA used in this study contained tissues from 46 lung cancer cases with information on LN metastasis. Examination of the expression profile of each antigen revealed that glucosidase II, unnamed protein product, glycyl-tRNA synthetase, chaperonin containing TCP1 subunit 8 and eukaryotic initiation factor 4AII were not expressed in the clinical samples of lung tumor tissues, suggesting that these proteins were only produced in the cancer cell lines. However, ten other proteins were expressed in the clinical samples of lung tumor tissues (Table III). Results summarized in Table III also show that the expression ratio of OSBPL5 and CALU, among all the expressed candidate proteins, were significantly higher in the $\mathrm{LN}$ metastasis-positive cases than in the metastasis-negative 
Table I. Quantitative analysis and identification of lung cancer related proteins by MALDI-TOF/MS.

\begin{tabular}{|c|c|c|c|c|c|}
\hline Spot & Protein name & Accesion no. & MW (kDa) & $\mathrm{pI}$ & $\begin{array}{c}\text { Expression ratio } \\
\text { RERF-LC-KJ/ } \\
\text { RERF-LC-MS }\end{array}$ \\
\hline$\# 1$ & Actinin $\alpha \mathrm{I}(\mathrm{ACTN} 1)$ & P12814 & 103 & 5.3 & 0.48 \\
\hline \#2 & Glucosidase II & CAA04006 & 107 & 5.7 & 3.2 \\
\hline \#3 & Oxysterol-binding protein-like 5 (OSBPL5) & Q9H0X9 & 98 & 5.9 & 2.2 \\
\hline \#4 & Unnamed protein product & CAA35893 & 69 & 5.9 & 2.4 \\
\hline \#5 & Glycyl-tRNA synthetase (GARS) & P41250 & 83 & 5.9 & 0.38 \\
\hline \#6 & Chaperonin containing TCP1 subunit 8 & Q53HU0 & 59 & 5.5 & 3.1 \\
\hline \#7 & Downstream of tyrosine kinase 7 (DOK7) & Q18PE1 & 53 & 6.4 & 0.40 \\
\hline \#8 & Aldehyde dehydrogenase $\left(\mathrm{NAD}^{+}\right)$ & CAA53176 & 51 & 5.8 & 2.7 \\
\hline \#9 & Cytokeratin 7 (CKT7) & P08729 & 51 & 5.4 & 3.9 \\
\hline \#10 & Cytokeratin 8 (CKT8) & P05787 & 53 & 5.5 & 6.6 \\
\hline \#11 & Calumenin (CALU) & O43852 & 38 & 4.5 & 2.6 \\
\hline \#12 & Eukaryotic initiation factor 4AII (EIF4A2) & Q14240 & 47 & 5.3 & 2.4 \\
\hline$\# 13$ & Cytokeratin 18 (CKT18) & P05783 & 47 & 5.3 & 5.8 \\
\hline \#14 & Acyl CoA dehydrogenase (ACD) & AAA74424 & 42 & 5.9 & 2.1 \\
\hline \#15 & Glutathione S-transferase P (GSTP1) & P09211 & 23 & 5.4 & 0.36 \\
\hline
\end{tabular}

Table II. Enrichment and isolation of scFv antibodies to identified proteins from scFv phage display libraries.

\begin{tabular}{llrrrr}
\hline & & \multicolumn{4}{c}{ Output/input ratio $\left(\times 10^{-8}\right)$} \\
\cline { 3 - 6 } Spot & \multicolumn{1}{c}{ Protein name } & 1st & 2nd & 3rd & 4 th \\
\hline \#1 & ACTN1 & 3 & 130 & 400 & 11,000 \\
$\# 2$ & Glucosidase II & 3 & 65 & 500 & 350 \\
$\# 3$ & OSBPL5 & 4 & 13 & 2,500 & 2,800 \\
$\# 4$ & Unnamed protein product & 3 & 6 & 130 & 6,000 \\
$\# 5$ & GARS & 9 & 16 & 170 & 4,350 \\
$\# 6$ & Chaperonin containing & 8 & 24 & 210 & 2,750 \\
& TCP1 subunit 8 & & & & \\
$\# 7$ & DOK7 & 12 & 40 & 150 & 2,150 \\
$\# 8$ & NAD & 15 & 12 & 100 & 2,300 \\
$\# 9$ & CKT7 & 5 & 12 & 70 & 1,450 \\
$\# 10$ & CKT8 & 9 & 140 & 150 & 21,000 \\
$\# 11$ & CALU & 16 & 240 & 60 & 2,000 \\
$\# 12$ & EIF4A2 & 23 & 21 & 77 & 3,500 \\
$\# 13$ & CKT18 & 7 & 2 & 170 & 350 \\
$\# 14$ & ACD & 35 & 6 & 37 & 4,500 \\
$\# 15$ & GSTP1 & 14 & 14 & 110 & 2,200 \\
\hline
\end{tabular}

cases $(\mathrm{p}=0.0156$ and 0.0055 , respectively). Moreover, 15 cases out of a total of 46 lung tumor cases were OSBPL5 and CALU double-positive, and 12 of them (80\% of OSBPL5 and CALU double-positive cases) were LN metastasis-positive. Therefore, the correlation analysis between protein expression and clinicopathological characteristic revealed significant association between OSBPL5 and CALU expression and LN metastasis in lung tumors.

Effects of OSBPL5 and CALU expression (overexpression or knockdown) on invasiveness of cells. To delineate the functions of OSBPL5 and CALU in metastatic lung cancer, we analyzed the effects of gene overexpression and gene knockdown on lung cancer cell invasiveness, a main characteristic of metastasis. First, we transfected RERF-LC-MS cells with either OSBPL5 expression plasmid pCMV-OSBPL5 or with CALU expression plasmid pCMV-CALU, and confirmed that OSBPL5 or CALU, respectively, was indeed overexpressed in these cells. Test results for the invasiveness of cells, as shown in Fig. 4A, clearly indicate that the RERF-LC-MS cells overexpressing either OSBPL5 or CALU were significantly more invasive than the cells transfected with the control plasmid. Next, we transfected RERF-LC-KJ cells with OSBPL5 siRNA or CALU siRNA and then examined the invasiveness of cells in which these genes were knocked down. As shown in Fig. 4B, the invasiveness of cells transfected with either OSBPL5 or CALU siRNA was significantly lower compared to that of the mock group, while there was no difference in cell proliferation (data not shown). Knockdown of expression of either OSBPL5 or CALU by transfection of RERF-LC-KJ cells with the respective siRNA did not diminish the invasiveness of cells completely, suggesting that the expression of OSBPL5 and CALU are probably partly responsible for the increased invasiveness of cells. An inhibitory effect of the cell invasiveness by OSBPL5 gene-knockdown was also observed in the invasive lung cancer cells (HARA-B), which 


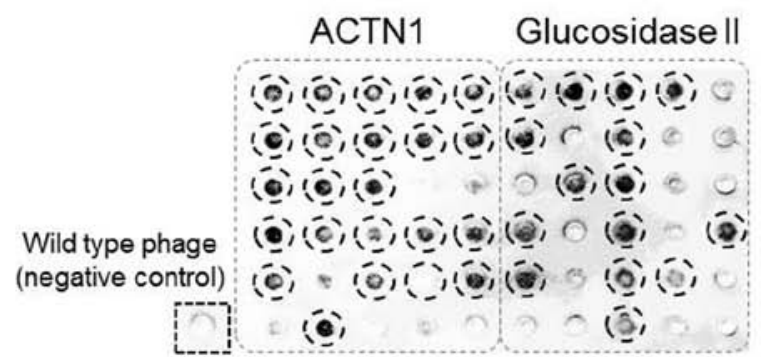

Positive rate $23 / 30$ clones $15 / 30$ clones

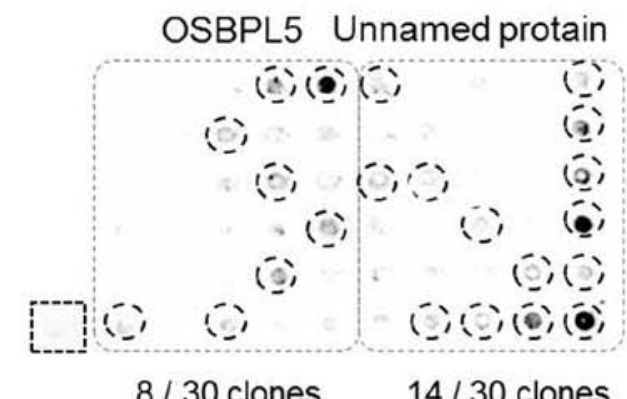

$8 / 30$ clones $\quad 14 / 30$ clones
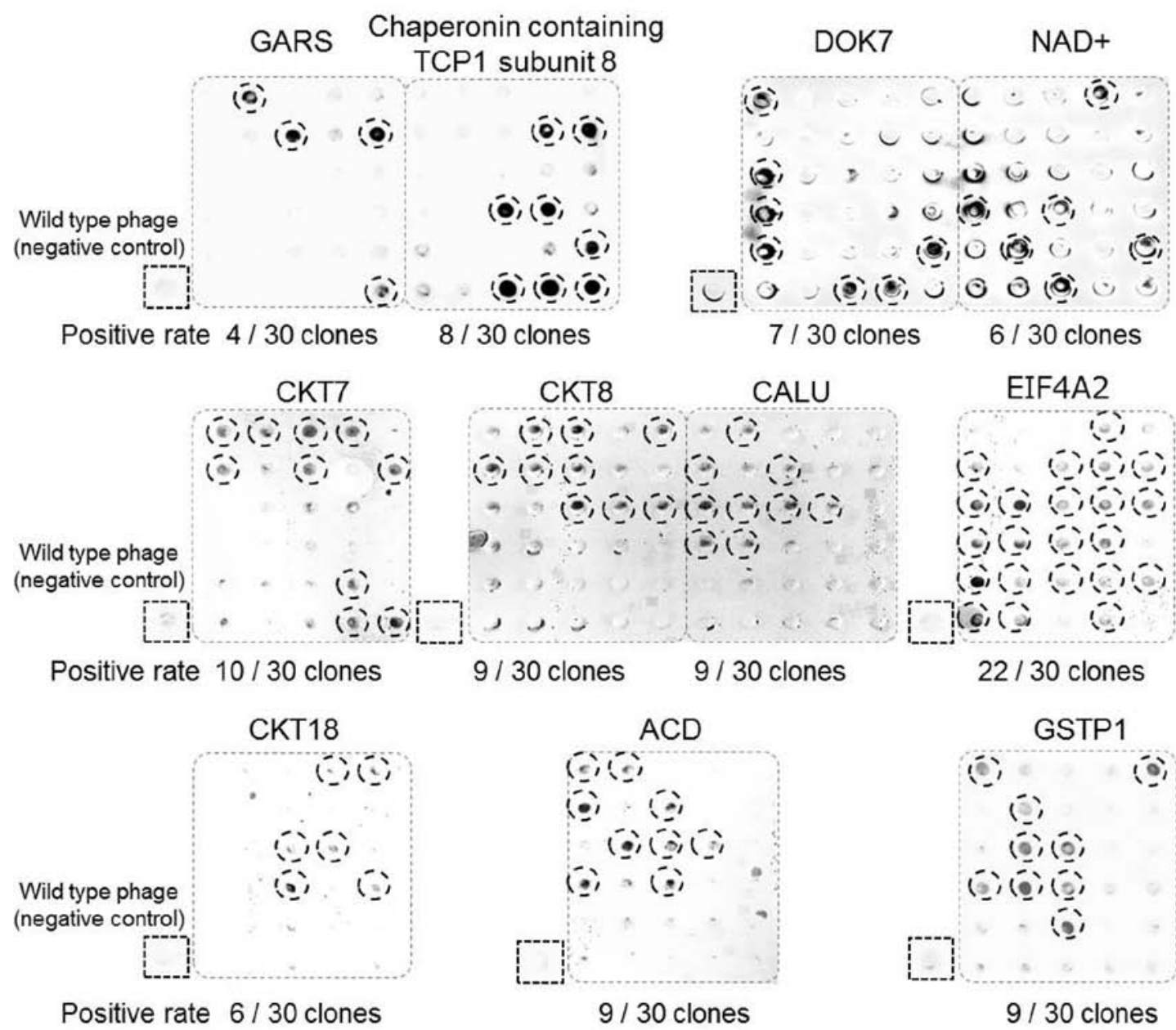

Figure 2. Enrichment of scFv antibodies to identified target proteins. Candidate scFv phage antibodies to identified proteins were enriched by four rounds of affinity panning. Binding characteristics of scFv phage antibodies were further evaluated by phage ELISA. For this purpose, phages purified from 30 individual clones (selected from a pool of preliminary clones/antigen) were incubated with the respective proteins blotted on nitrocellulose membranes using a dot blot apparatus as described above. Binding of each scFv antibody to the respective antigen was detected using HRP-conjugated anti-M13 monoclonal antibody. Wild-type phage was used as a negative control. Positive clones are marked using dotted circles.

were derived from a bone lesion formed after the intracardiac inoculation of HARA cells (Fig. 5). These results suggested that OSBPL5 and CALU might play a critical role in facilitating invasiveness of lung cancer cells.

Expression analysis of OSBPL5 and CALU in normal lung tissues. To further determine the usefulness of OSBPL5 and CALU as diagnostic or therapeutic targets, we analyzed the expression levels of these proteins in normal lung tissues. TMA analysis using lung cancer and normal lung tissues showed that OSBPL5 and CALU were specifically expressed in the lung tumor tissues (Table IV). Our observation that both OSBPL5 and CALU were specifically expressed in the lung tumor tissues suggested that these two proteins might have some functional roles in lung cancer cells. Thus, they could either help in elucidating the underlying mechanism of lung cancer or serve as targets for developing therapies against lung cancer.

\section{Discussion}

In this study, we successfully identified OSBPL5 and CALU as metastasis-related proteins in lung tumors, which were highly expressed in metastasis-positive cases and facilitated inva- 


\section{Blotted antigen}
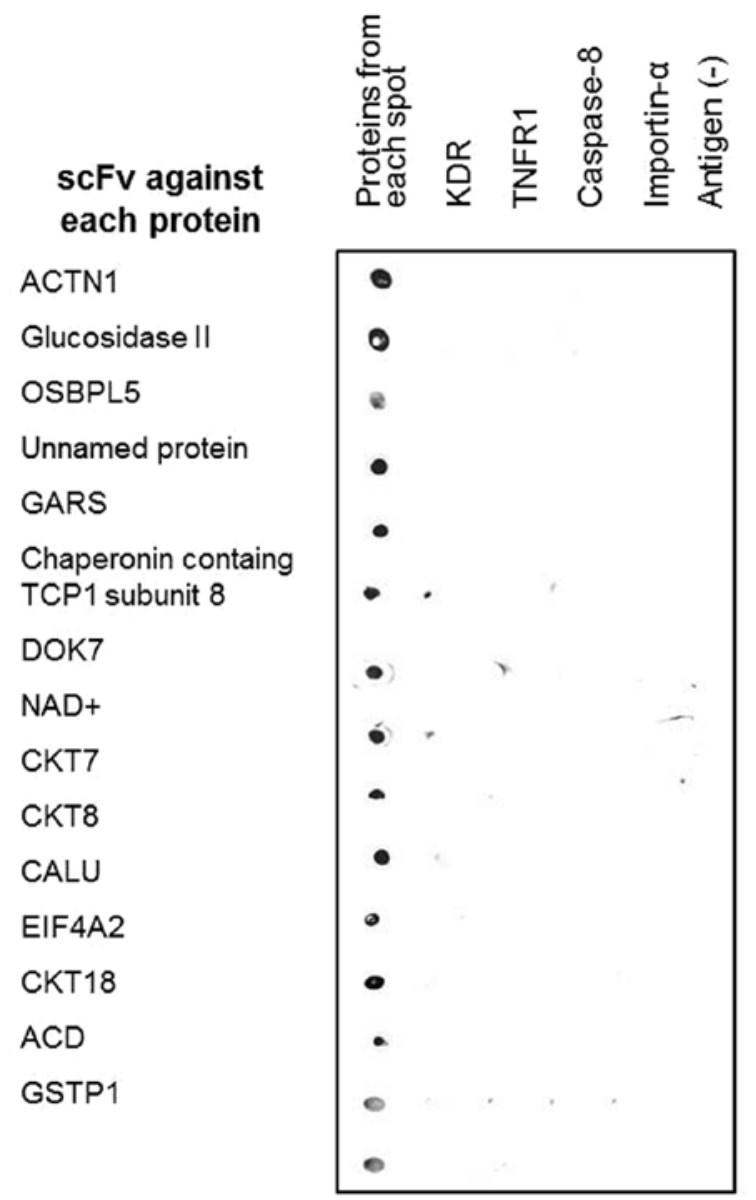

Figure 3. Specificity of scFv phage antibodies. Only clones showing highest affinities to respective target proteins were selected for the specificity evaluation. The specificity of a phage antibody clone was analyzed by examining its binding to various other non-specific antigens, namely KDR, TNFR 1 , caspase- 8 and importin- $\alpha$. Each phage antibody was purified and then equal amount of the purified phage was incubated with the indicated antigen. Binding of the antibody to antigen was assessed using HRP-conjugated antiM13 monoclonal antibody.

siveness of lung cancer cells. This was achieved by carefully selecting the target proteins from a large pool of differentiallyexpressed proteins rapidly and efficiently.

OSBPL5 is a member of the oxysterol binding protein (OSBP) family (24). OSBP is a cytosolic mammalian protein that binds to an oxysterol ligand and interacts with the golgi membrane and is involved in vesicle transport, lipid metabolism, and signal transduction. Previous studies suggested that metabolism-related molecules were associated with LN metastasis, for example, association of angiopoietin-like protein 4 (25) or acid phosphatase 6 (26) in esophageal squamous cell carcinoma and heart-type fatty acid-binding protein in gastric carcinoma (27). It was also shown that statins, inhibitors of 3-hydroxy-3-metylglutaryl coenzyme A (HMG-CoA) reductase, inhibited metastasis (28). These results suggested that OSBPL5, a metabolism-related molecule, might be involved in metastasis. Consistent with this notion, it was reported earlier that OSBPL5 expression is related to invasion and poor prognosis of pancreatic cancer $(29,30)$. Thus,
Table III. Correlation analysis between expression profile and lymph node metastasis.

\begin{tabular}{lcc}
\hline & \multicolumn{2}{c}{$\begin{array}{c}\text { Ratio of candidate } \\
\text { protein-positive cases }\end{array}$} \\
\cline { 2 - 3 } Protein name & $\begin{array}{c}\text { In lymph node } \\
\text { metastasis-negative } \\
\text { cases }(20 \text { cases) }(\%)\end{array}$ & $\begin{array}{c}\text { In lymph node } \\
\text { metastasis-positive } \\
\text { cases (26 cases) (\%) }\end{array}$ \\
\hline ACTN1 & $4 / 20(20)$ & $4 / 26(15)$ \\
OSBPL5 & $4 / 20(20)$ & $15 / 26(58)$ \\
DOK7 & $17 / 20(85)$ & $13 / 26(50)$ \\
NAD & $5 / 20(25)$ & $7 / 26(27)$ \\
CKT7 & $14 / 20(70)$ & $10 / 26(38)$ \\
CKT8 & $19 / 20(95)$ & $21 / 26(81)$ \\
CALU & $3 / 20(15)$ & $15 / 26(58)$ \\
CKT18 & $15 / 20(75)$ & $13 / 26(50)$ \\
ACD & $8 / 20(40)$ & $6 / 26(23)$ \\
GSTP1 & $10 / 20(50)$ & $11 / 26(42)$ \\
\hline
\end{tabular}

OSBPL5 may play a role in facilitating metastasis of lung cancer, similar to that suggested for pancreas cancer.

CALU is a calcium binding protein in the endoplasmic reticulum (ER) and is involved in such ER functions as protein folding and sorting (31). It has been reported earlier that heat shock proteins were associated with LN metastasis $(32,33)$, suggesting that chaperones such as CALU could also play a role in metastasis. Unlike OSBPL5, CALU was found to be downregulated in cancer cell lines with high metastatic potential and were found in head and neck (34), as well as in liver cancer (35). The function of CALU in lung cancer may, however, be different from that in head and neck cancer and liver cancer.

In the cases where both proteins were expressed, $80 \%$ were found to be LN metastasis-positive cases. Moreover, these proteins were expressed only in lung tumor tissues, not in normal lung tissues. Therefore, these findings suggested that they could be promising targets for accurate diagnosis and prediction of metastasis; however, further experiments, such as a prospective study, are required. Furthermore, gene knockdown experiments showed that knocking down the expression of OSBPL5 or CALU inhibited invasiveness of lung cancer cells. These results suggested that OSBPL5 and CALU might also be considered as useful target proteins for metastasis therapy, although further experiments, such as their biodistribution analyses and therapeutic experiments, are needed.

In conclusion, by using an antibody proteomics technology, we identified OSBPL5 and CALU as metastasisrelated proteins in lung tumors. Furthermore, we have revealed that OSBPL5 and CALU promoted the invasiveness of lung cancer cells. We hope that the data presented would contribute to the elucidation of molecular mechanism of metastasis and help in developing diagnosis markers and drugs against metastasis in lung cancer. 
A

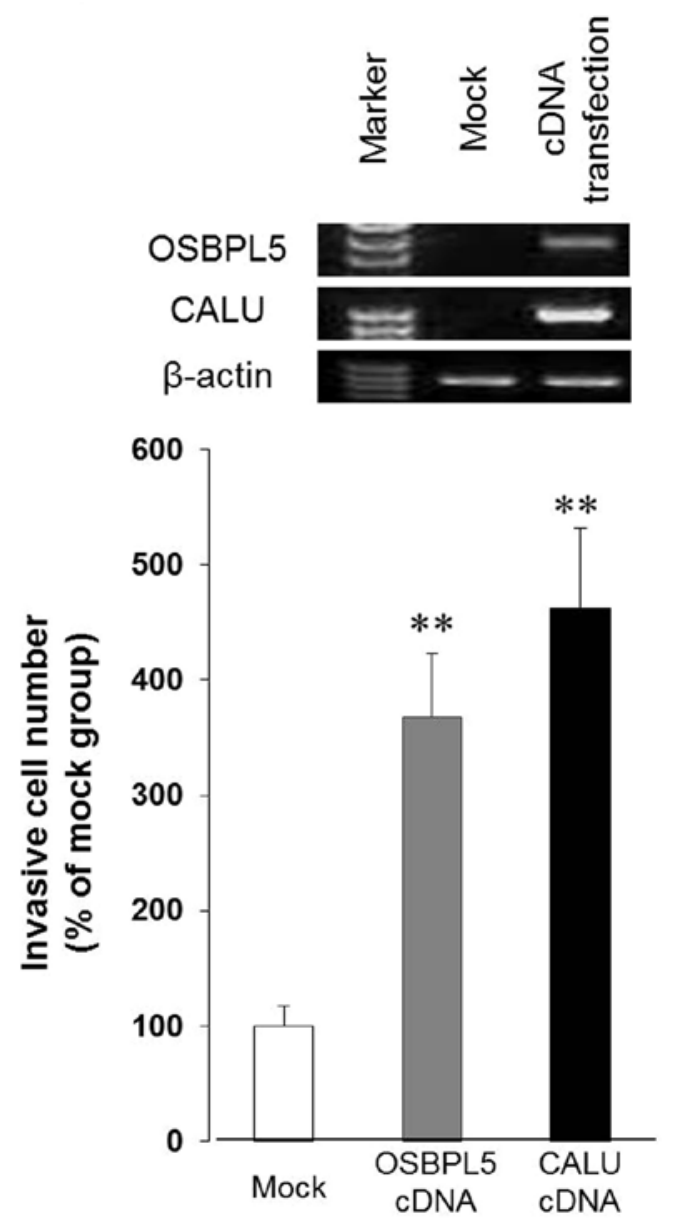

B
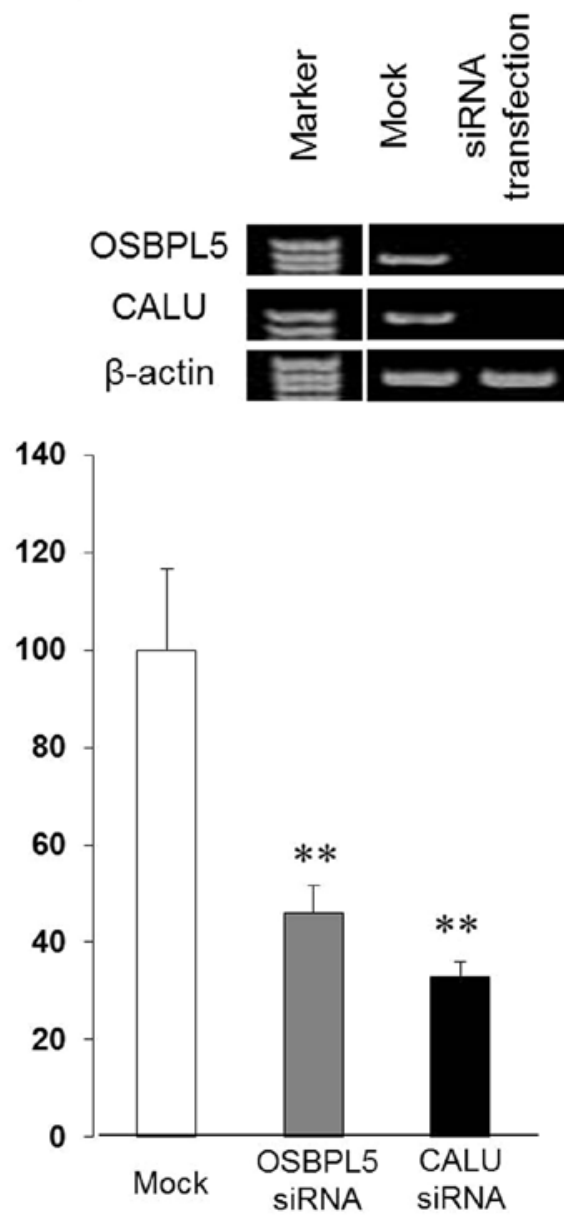

Figure 4. Effect of OSBPL5 and CALU expression on invasiveness of lung cancer cells. The invasiveness of lung cancer cells was analyzed using a 96-well BME cell invasion assay kit as described in Materials and methods. (A) RERF-LC-MS cells transfected with OSBPL5 or CALU cDNA expression plasmid. Upper panel, RT-PCR analysis of OSBPL5 and CALU expression in RERF-LC-MS cells transfected with the indicated cDNA plasmid. Lower panel, invasiveness of RERF-LC-MS cells transfected with the indicated cDNA plasmid or invasiveness of mock cells. (B) Upper panel, RT-PCR analysis of OSBPL5 and CALU expression in RERF-LC-KJ cells transfected with the indicated siRNA. Lower panel, invasiveness of RERF-LC-KJ cells transfected with the indicated siRNA and invasiveness of the mock cells. Error bars represent mean \pm SD of triplicate assays ${ }^{* *} \mathrm{p}<0.01$ vs control).

A HARA-B cells

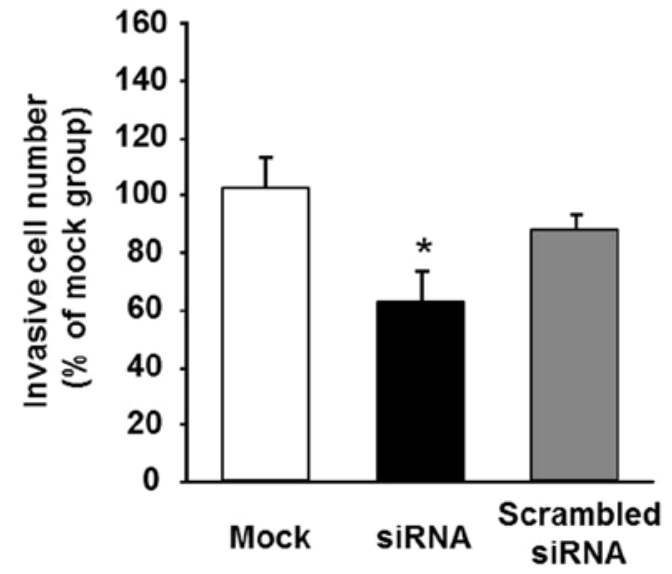

B HARA cells

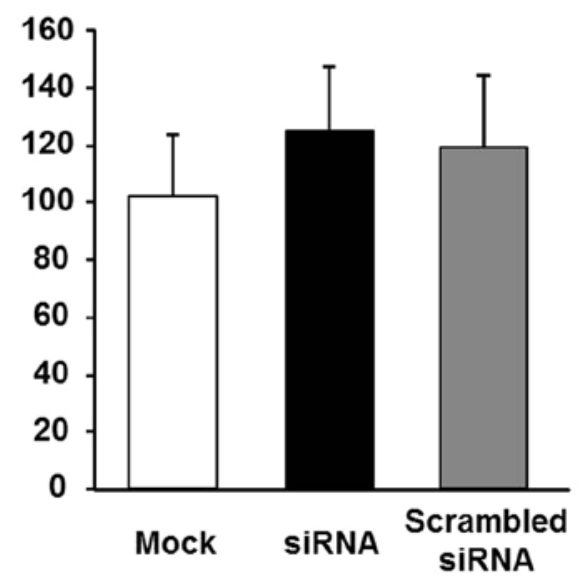

Figure 5. Relationship between the expression level of OSBPL5 and cell invasive activity in HARA and HARA-B cells. (A and B) Knockdown of OSBPL5 gene with siRNA and invasiveness of cells. HARA-B (A) and HARA (B) cells were transfected with the indicated siRNA, cells were then seeded into the upper chamber of the 96-well cell culture inserts of the BME cell invasion assay kit as described in Materials and methods and incubated for $72 \mathrm{~h}$. The number of transfected cells found in the bottom chamber was counted and plotted: control cells (open bar), OSBPL5 siRNA tranfected cells (black bar) and scrambled siRNA transfected cells (gray bar). Error bars represent mean \pm SD of triplicate assays ( $\mathrm{P}<0.05$ vs control). 
Table IV. Microarray analysis of lung cancer and normal tissues using scFv-expressing phages against candidate proteins.

\begin{tabular}{llr}
\hline & \multicolumn{3}{c}{$\begin{array}{c}\text { Expression ratio of } \\
\text { each candidate }\end{array}$} \\
\cline { 2 - 3 } Protein name & $\begin{array}{c}\text { Normal lung } \\
\text { tissues }(\%)\end{array}$ & $\begin{array}{c}\text { Lung cancer } \\
\text { tissues }(\%)\end{array}$ \\
\hline ACTN1 & $2 / 9(22)$ & $8 / 50(16)$ \\
Glucosidase II & $0 / 9(0)$ & $0 / 50(0)$ \\
OSBPL5 & $0 / 9(0)$ & $22 / 50(44)$ \\
Unnamed protein product & $0 / 9(0)$ & $0 / 50(0)$ \\
GARS & $0 / 9(0)$ & $0 / 50(0)$ \\
Chaperonin containing & $0 / 9(0)$ & $0 / 50(0)$ \\
TCP1 subunit 8 & & \\
DOK7 & $3 / 9(33)$ & $30 / 50(60)$ \\
NAD & & $12 / 50(24)$ \\
CKT7 & $0 / 9(0)$ & $24 / 50(48)$ \\
CKT8 & $2 / 9(22)$ & $40 / 50(80)$ \\
CALU & $0 / 9(0)$ & $20 / 50(40)$ \\
EIF4A2 & $0 / 9(0)$ & $0 / 50(0)$ \\
CKT18 & $0 / 9(0)$ & $28 / 50(56)$ \\
ACD & $0 / 9(0)$ & $14 / 50(28)$ \\
GSTP1 & $1 / 9(11)$ & $21 / 50(42)$ \\
\hline
\end{tabular}

\section{Acknowledgements}

This study was supported in part by Grants-in-Aid for Scientific Research from the Project for Development of Innovative Research on Cancer Therapeutics, the Ministry of Education, Culture, Sports, Science and Technology of Japan, and from the Japan Society for the Promotion of Science. This study was also supported in part by Health Labor Sciences Research Grants from the Ministry of Health, Labor and Welfare of Japan.

\section{References}

1. Parkin DM, Bray F, Ferlay $\mathbf{J}$ and Pisani P: Global cancer statistics, 2002. CA Cancer J Clin 55: 74-108, 2005.

2. Steeg PS: Metastasis suppressors alter the signal transduction of cancer cells. Nat Rev Cancer 3: 55-63, 2003.

3. Rochefort H, Capony F and Garcia M: Cathepsin D: A protease involved in breast cancer metastasis. Cancer Metastasis Rev 9: 321-331, 1990

4. Zheng S, Chang Y, Hodges KB, Sun Y, Ma X, Xue Y, Williamson SR, Lopez-Beltran A, Montironi R and Cheng L: Expression of KISS1 and MMP-9 in non-small cell lung cancer and their relations to metastasis and survival. Anticancer Res 30: 713-718, 2010.

5. Koga F, Kihara K and Neckers L: Inhibition of cancer invasion and metastasis by targeting the molecular chaperone heat-shock protein 90. Anticancer Res 29: 797-807, 2009.

6. Tsutsumi S and Neckers L: Extracellular heat shock protein 90: A role for a molecular chaperone in cell motility and cancer metastasis. Cancer Sci 98: 1536-1539, 2007.

7. Kang Y and Massagué J: Epithelial-mesenchymal transitions: Twist in development and metastasis. Cell 118: 277-279, 2004.

8. Tsuji T, Ibaragi S and Hu GF: Epithelial-mesenchymal transition and cell cooperativity in metastasis. Cancer Res 69: 7135-7139, 2009.
9. Lee GH, Yan C, Shin SJ, Hong SC, Ahn T, Moon A, Park SJ Lee YC, Yoo WH, Kim HT, et al: BAX inhibitor-1 enhances cancer metastasis by altering glucose metabolism and activating the sodium-hydrogen exchanger: The alteration of mitochondrial function. Oncogene 29: 2130-2141, 2010.

10. Torosian M, Charland S and Lappin J: Biochemical modulation of tumor-growth, metastasis and host metabolism. Oncol Rep 2: $1141-1145,1995$.

11. Hanash S: Disease proteomics. Nature 422: 226-232, 2003.

12. Imai S, Nagano K, Yoshida Y, Okamura T, Yamashita $T$, Abe Y, Yoshikawa T, Yoshioka Y, Kamada H, Mukai Y, et al: Development of an antibody proteomics system using a phage antibody library for efficient screening of biomarker proteins. Biomaterials 32: 162-169, 2011.

13. Yamashita T, Nagano K, Kanasaki S, Maeda Y, Furuya T, Inoue M, Nabeshi H, Yoshikawa T, Yoshioka Y, Itoh N, et al: Annexin A4 is a possible biomarker for cisplatin susceptibility of malignant mesothelioma cells. Biochem Biophys Res Commun 421: 140-144, 2012.

14. Yamashita T, Okamura T, Nagano K, Imai S, Abe Y, Nabeshi H, Yoshikawa T, Yoshioka Y, Kamada H, Tsutsumi Y, et al: Rho GDP-dissociation inhibitor alpha is associated with cancer metastasis in colon and prostate cancer. Pharmazie 67: 253-255, 2012.

15. Yoshida $Y$, Yamashita T, Nagano K, Imai S, Nabeshi $H$, Yoshikawa T, Yoshioka Y, Abe Y, Kamada H, Tsutsumi Y, et al: Limited expression of reticulocalbin-1 in lymphatic endothelial cells in lung tumor but not in normal lung. Biochem Biophys Res Commun 405: 610-614, 2011.

16. Nagano K, Kanasaki S, Yamashita T, Maeda Y, Inoue M, Higashisaka K, Yoshioka Y, Abe Y, Mukai Y, Kamada H, et al: Expression of Eph receptor A10 is correlated with lymph node metastasis and stage progression in breast cancer patients. Cancer Med 2: 972-977, 2013.

17. Nagano K, Maeda Y, Kanasaki S, Watanabe T, Yamashita T, Inoue M, Higashisaka K, Yoshioka Y, Abe Y, Mukai Y, et al: Ephrin receptor A10 is a promising drug target potentially useful for breast cancers including triple negative breast cancers. J Control Release 189: 72-79, 2014.

18. Nagano K, Yamashita T, Inoue M, Higashisaka K, Yoshioka Y, Abe Y, Mukai Y, Kamada H, Tsutsumi Y and Tsunoda S: Eph receptor A10 has a potential as a target for a prostate cancer therapy. Biochem Biophys Res Commun 450: 545-549, 2014.

19. Imai S, Mukai Y, Nagano K, Shibata H, Sugita T, Abe Y, Nomura T, Tsutsumi Y, Kamada H, Nakagawa S, et al: Quality enhancement of the non-immune phage scFv library to isolate effective antibodies. Biol Pharm Bull 29: 1325-1330, 2006.

20. Teraoka S, Kyoizumi S, Seyama T, Yamakido M and Akiyama M: Scid mice model for the in-vivo study of human oncotherapy studies on the growth and metastasis of human lung-cancer. Int $\mathrm{J}$ Oncol 5: 501-508, 1994.

21. Teraoka S, Kyoizumi S, Seyama T, Yamakido M and Akiyama M: A novel SCID mouse model for studying spontaneous metastasis of human lung cancer to human tissue. Jpn J Cancer Res 86: 419-423, 1995.

22. Hirai K, Shimada H, Ogawa T and Taji S: The spread of human lung cancer cells on collagens and its inhibition by type III collagen. Clin Exp Metastasis 9: 517-527, 1991.

23. Kuramochi M, Fukuhara H, Nobukuni T, Kanbe T, Maruyama T, Ghosh HP, Pletcher M, Isomura M, Onizuka M, Kitamura T, et al: TSLC1 is a tumor-suppressor gene in human non-small-cell lung cancer. Nat Genet 27: 427-430, 2001.

24. Fairn GD and McMaster CR: Emerging roles of the oxysterolbinding protein family in metabolism, transport, and signaling. Cell Mol Life Sci 65: 228-236, 2008.

25. Shibata K, Nakayama T, Hirakawa H, Hidaka S and Nagayasu T: Clinicopathological significance of angiopoietin-like protein 4 expression in oesophageal squamous cell carcinoma. J Clin Pathol 63: 1054-1058, 2010.

26. Ando T, Ishiguro H, Kuwabara Y, Kimura M, Mitsui A, Kurehara H, Sugito N, Tomoda K, Mori R, Takashima N, et al: Expression of ACP6 is an independent prognostic factor for poor survival in patients with esophageal squamous cell carcinoma. Oncol Rep 15: 1551-1555, 2006.

27. Hashimoto T, Kusakabe T, Sugino T, Fukuda T, Watanabe K, Sato Y, Nashimoto A, Honma K, Kimura H, Fujii H, et al: Expression of heart-type fatty acid-binding protein in human gastric carcinoma and its association with tumor aggressiveness, metastasis and poor prognosis. Pathobiology 71: 267-273, 2004 . 
28. Hindler K, Cleeland CS, Rivera E and Collard CD: The role of statins in cancer therapy. Oncologist 11: 306-315, 2006.

29. Ishikawa S, Nagai Y, Masuda T, Koga Y, Nakamura T, Imamura $Y$, Takamori H, Hirota M, Funakosi A, Fukushima M, et al: The role of oxysterol binding protein-related protein 5 in pancreatic cancer. Cancer Sci 101: 898-905, 2010.

30. Koga Y, Ishikawa S, Nakamura T, Masuda T, Nagai Y, Takamori $H$, Hirota M, Kanemitsu K, Baba Y and Baba H: Oxysterol binding protein-related protein-5 is related to invasion and poor prognosis in pancreatic cancer. Cancer Sci 99: 2387-2394, 2008

31. Honoré B: The rapidly expanding CREC protein family: Members, localization, function, and role in disease. BioEssays 31: 262-277, 2009.

32. Cappello F, David S, Rappa F, Bucchieri F, Marasà L, Bartolotta TE, Farina F and Zummo G: The expression of HSP60 and HSP10 in large bowel carcinomas with lymph node metastase. BMC Cancer 5: 139, 2005.
33. Castilla C, Congregado B, Conde JM, Medina R, Torrubia FJ, Japón MA and Sáez C: Immunohistochemical expression of Hsp60 correlates with tumor progression and hormone resistance in prostate cancer. Urology 76: 1017.e1-6, 2010.

34. Wu W, Tang X, Hu W, Lotan R, Hong WK and Mao L: Identification and validation of metastasis-associated proteins in head and neck cancer cell lines by two-dimensional electrophoresis and mass spectrometry. Clin Exp Metastasis 19: 319-326, 2002.

35. Ding SJ, Li Y, Shao XX, Zhou H, Zeng R, Tang ZY and Xia QC: Proteome analysis of hepatocellular carcinoma cell strains, MHCC97-H and MHCC97-L, with different metastasis potentials. Proteomics 4: 982-994, 2004 